\title{
Influence of loading-rate and steel fibers on the shear strength of ultra high performance concrete
}

\author{
Lukic Bratislav and Forquin Pascal ${ }^{\mathrm{a}}$ \\ Laboratoire 3SR, Grenoble Alpes University, BP. 53, 38041 Grenoble Cedex 9, France
}

\begin{abstract}
The paper describes quasi-static and dynamic experimental methods used to examine the confined shear strength of an Ultra High Performance Concrete, with and without the presence of steel fibers in the concrete composition. An experimental setup was created to investigate the concrete shear strength under quasi-static loading regime using a hydraulic press Schenk while dynamic shear strength was characterized by subjecting concrete samples to dynamic loading through a modified Split Hopkinson Pressure Bar. Both methods are based on a Punch Through Shear (PTS) test with a well-instrumented aluminum passive confinement ring that allows measuring the change of radial stress in the shear ligament throughout the test. Firstly, four equally distributed radial notches have been performed in order to deduce the radial stress by suppressing a self-confinement of the sample peripheral part. However, by analyzing the strain gauge data from the confinement ring, it has been noticed that these were apparently insufficient, especially for fiber-reinforced samples, resulting in subsequently practicing eight radial notches through the sample peripheral part. The results obtained from both procedures are reported and discussed.
\end{abstract}

\section{Introduction}

Concrete structures such as nuclear power plants, dams, bridges or bunkers can be exposed to intensive loadings such as earthquakes, blasting or projectile-impact. During these dynamic loading conditions, in addition to tensile failure, shear damage modes can be observed as cratering on the front face and shear strain close to the crater [1]. Mode II shear fracturing has also been observed in ultra-high performance concrete during edge-on impact experiments [2]. However, the shear strength of ultra-high performance concrete remains unknown and the influence of steel fibers as well as strain-rate on the shear strength need to be investigated. The Punch Through Shear (PTS) experimental technique was first proposed by Watkins [3]. The cylindrical specimen contains two cylindrical notches on the top and the bottom face such that the inner diameter of the upper notch coincides with the outer diameter of the lower notch consequently forming a cylindrical shear surface. In further works, the specimen was first subjected to a pure hydrostatic pressure whereas an axial load was added in the second step to create a shear fracturing of the ligament. This technique was more recently used by Montenegro [4] to evaluate the fracture energy of conventional concrete (maximum aggregate size $6 \mathrm{~mm}$ ) as function of the hydrostatic pressure. In the present work, quasi-static and dynamic Punch Through Shear (PTS) tests have been conducted with an ultra-high strength concrete, with and without short steel fiber reinforcement.

A well-instrumented passive confining ring has been applied to the PTS samples in a similar way than in quasi-oedometric compression tests [5] allowing to deduce radial stress in the fractured zone. An overview of the two experimental procedures is given with presenting and discussing the results obtained.

\footnotetext{
${ }^{a}$ Corresponding author: pascal. forquin@3sr-grenoble.fr
}

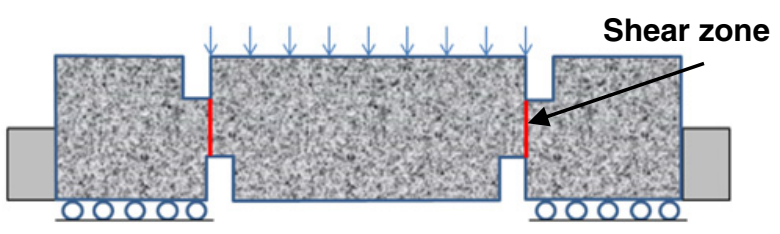

Figure 1. Punch Through Shear (PTS) specimen: Geometry of the sample used.

\section{Concrete sample}

\subsection{The PTS sample}

The specimen geometry is shown in the Fig. 1, inner diameter of the upper notch and outer diameter of the bottom notch coincide to form a straight cylindrical fracture surface. The length of the ligament ensures an almost perfectly homogeneous shear stress field in the shear zone. Moreover, the confining ring is decentered with respect to the specimen symmetry plane as to obtain uniform pressure field between the confining ring and the concrete sample and allows deducing the radial stress in the shear zone. The aluminum confining ring used in both experimental procedures is $10 \mathrm{~mm}$ in thickness and $15 \mathrm{~mm}$ in height and is instrumented with two strain gauges. The sample is carefully introduced into the confinement ring and the gap between the sample and the confining ring is filled by a bi-component resin $\left(\right.$ Chrysor $\left.{ }^{\circledR}\right)$. Furthermore, equally distributed radial notches are performed through the peripheral part of the specimen in order to avoid selfconfinement.

\subsection{Tested material}

The material tested is an Ultra High Performance Concrete (UHPC) whose mechanical properties and composition are gathered in Table 1. In contrast to conventional concrete, 
Table 1. Material Composition and Mechanical Properties of an Ultra High Performance Concrete [6].

\begin{tabular}{|l|c|c|}
\hline Composition & Ductal ${ }^{\circledR}$ \\
\hline Cement & {$\left[\mathrm{kg} / \mathrm{m}^{3}\right]$} & 730 \\
Silica Fume & {$\left[\mathrm{kg} / \mathrm{m}^{3}\right]$} & 235 \\
Crushed quartz grains & {$\left[\mathrm{kg} / \mathrm{m}^{3}\right]$} & 220 \\
Sand & {$\left[\mathrm{kg} / \mathrm{m}^{3}\right]$} & 885 \\
Superplasticizer & {$\left[\mathrm{kg} / \mathrm{m}^{3}\right]$} & 10 \\
Water & {$\left[1 / \mathrm{m}^{3}\right]$} & 160 \\
Steel fibers & {$[\mathrm{vol} . \%]$} & $2 \%$ \\
W/(C+SF) & 0.17 \\
\hline Mechanical Properties \\
\hline Density \\
Young's Modulus & {$\left[\mathrm{kg} / \mathrm{m}^{3}\right]$} & 2396 \\
Compressive strength & {$[\mathrm{GPa}]$} & 55 \\
\hline
\end{tabular}

the mixture contains finely crushed quartz grains and silica fume. The optimized granular mixture of UHPC, where the maximal grain size is approximately $0.6^{\circ} \mathrm{mm}$ (sand), and extremely low water to cementious material (cement and silica fumes) ratio allow casting a finely graded and highly homogeneous concrete matrix with outstanding mechanical properties. The use of superplasticizer in order to increase the workability is of great importance. In the present work, two specimen categories have been considered for shear testing: one which contains $2 \%$ volumetric ratio of short steel fibers $\left(0.2^{\circ} \mathrm{mm}\right.$ diameter and $7^{\circ} \mathrm{mm}$ in length) and the other without additional fiber reinforcement.

\section{Quasi-static shear testing}

\subsection{Principle of the experimental procedure}

The quasi-static experiments have been conducted in 3SR Laboratory. The PTS sample was placed on a steel support cylinder, ensuring a perfectly flat contact surface such that the driving force is equally distributed throughout the peripheral part of the sample. The inner diameter of the support cylinder corresponds to the outer diameter of specimen bottom notch, allowing the axial displacement of sample middle part with respect to its peripheral part. Additionally, a compression cylinder is attached to the sample upper so that its diameter coincides with the inner diameter of the upper notch. A sketch of the sample configuration cross section is shown in (b), Fig. 2a. The sample is then placed in The Schenk press, (b) Fig. 2b, whose capacity of $100 \mathrm{t}$ is more than sufficient for the purpose of the quasi-static testing. Two perfectly synchronized linear variable differential transformers (LVDTs) were used for measuring the displacement between press pressure caps. In order to achieve a desirably low strain-rate that corresponds to quasi-static loading regime, the load speed was set to $0.1 \mu \mathrm{m} / \mathrm{s}$, which corresponds approximately to rate of strain less than $10^{-4} \mathrm{~s}^{-1}$.

The test was driven until the value of displacement was equal to $10 \mathrm{~mm}$, for which it is considered that the total

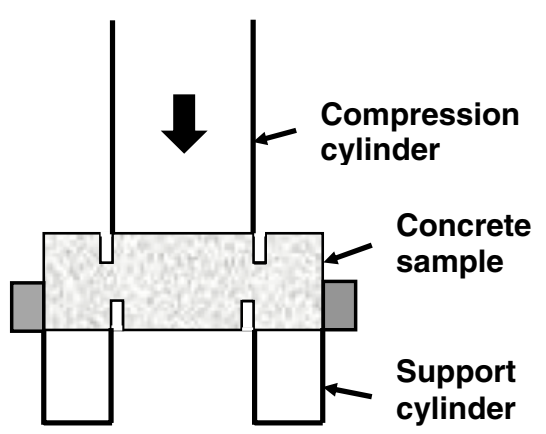

(a)

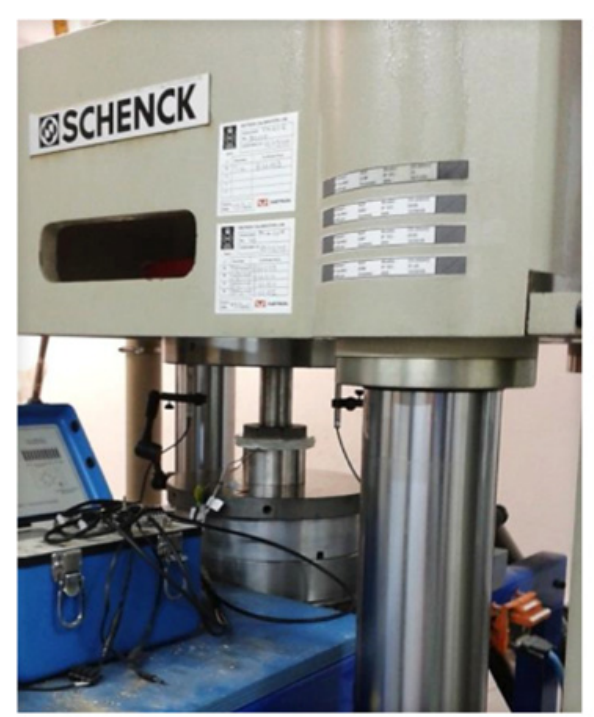

(b)

Figure 2. Quasi-static experimental set-up used in 3SR Laboratory (a) sketch of the PTS sample configuration, (b) the Schenk press.

failure of the shear ligament is reached. The mean shear stress in the shear ligament is deduced directly from the axial load that is transmitted onto the shear surface:

$$
\sigma_{\text {shear }}=\frac{F_{\text {axial }}}{S_{\text {ligement }}} .
$$

Furthermore, as reported in [7], the mean radial stress in the ligament is deduced from the average contact pressure between the sample and the confinement cell, knowing the contact surface and the area of the shear zone:

$$
\sigma_{\text {radial }}=\frac{S_{\text {contact }}}{S_{\text {ligament }}} P_{\text {contact }} \text {. }
$$

The contact pressure between the sample and the cell was obtained in a similar way as in the quasi-oedometric tests presented in [5], by post processing the data from the strain gauges glued on the aluminum confinement ring.

\subsection{Experimental results}

A series of PTS experiments have been carried out on Ultra High Performance Concrete (UHPC) with and without addition of short steel fibers. In order to skirt around 


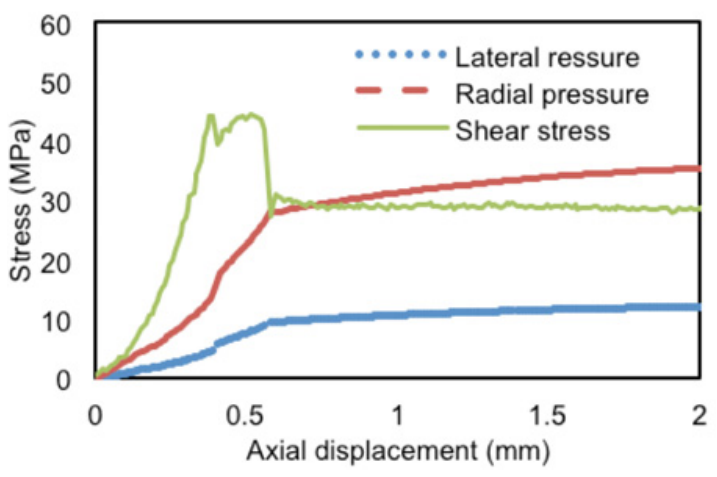

(a)

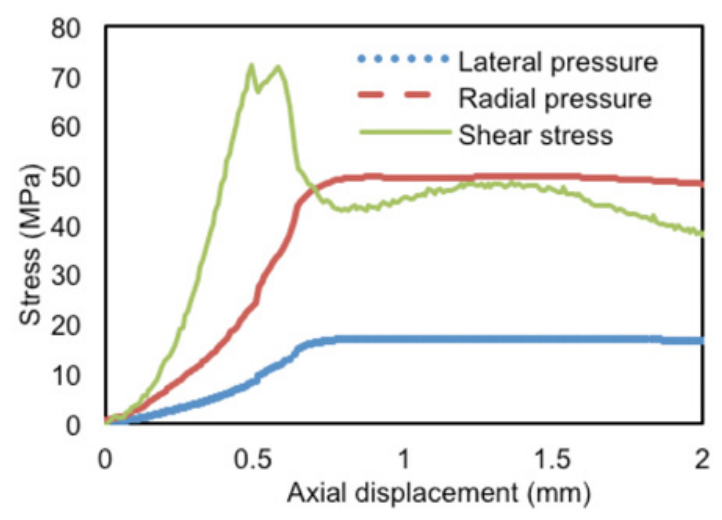

(b)

Figure 3. Results of quasi-static shear tests performed on fournotch specimen: (a) UHPC without additional steel fibers and (b) FUHPC with addition of short steel fibers (2\% volumetric content).

the self-confinement of the peripheral part, four equally distributed radial notches have been performed. The results of the quasi-static shear tests are reported in Fig. 3.

The experimental data indicates a maximum shear stress in specimen without addition of steel fibers of $45 \mathrm{MPa}$ while on the other hand the sample with $2 \%$ volumetric fiber reinforcement exhibited a markedly higher shear stress of about $70 \mathrm{MPa}$. A similar radial stress at the peak shear stress of about $25 \mathrm{MPa}$ is observed in both tests.

As the question raised from analyzing the four-notched quasi-static results was weather the increase of shear strength is induced solely by the presence of steel fibers in the shear zone or by the influence of an additional selfconfinement (both series of samples exhibited tensile radial cracking pattern), further experiments have been carried out on samples with eight peripheral radial notches and the results are reported in Fig. 4. The maximum shear stresses reached, in this case, were remarkably similar for both concrete mixtures, non-fibered sample showed shear strength of $42 \mathrm{MPa}$, while the reinforced sample exhibited shear stress of $49 \mathrm{MPa}$.

\subsection{Discussion on quasi-static results}

A double peak stress response is observed in all tested samples. At the first peak, a Mode I fracturing is expected, which is then followed by pure Mode II cracking at the second stress peak. A noticeable softening behaviour after

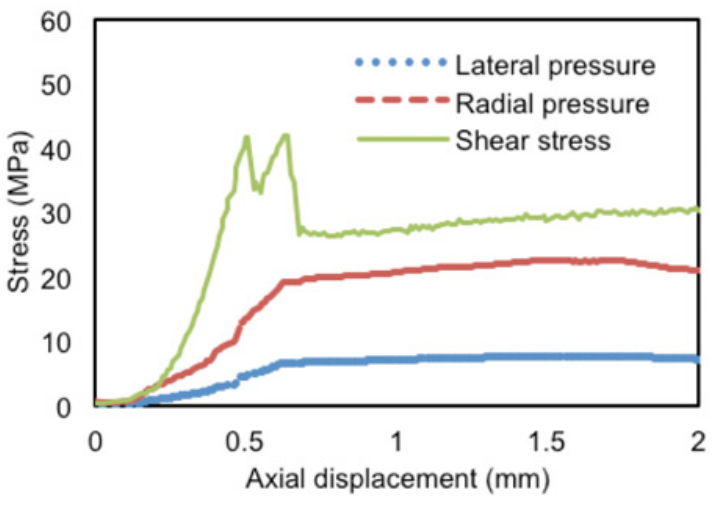

(a)

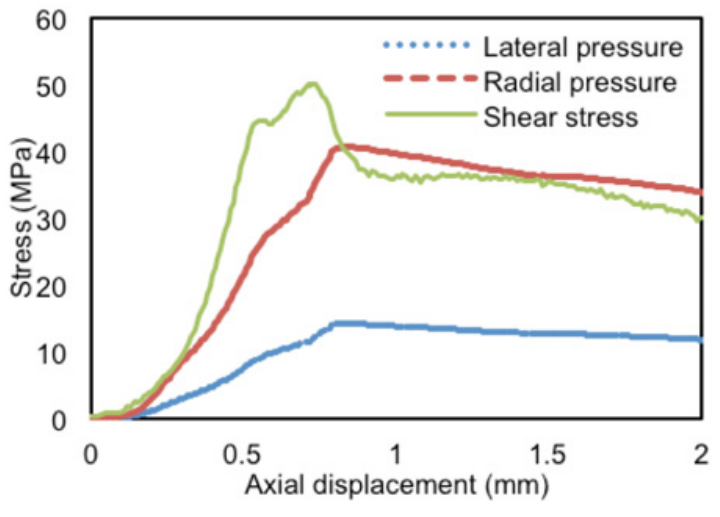

(b)

Figure 4. Results of quasi-static shear tests performed on eightnotch specimen: (a) UHPC without additional fibers and (b) UHPC with addition of $2 \%$ volumetric addition of short steel fibers.

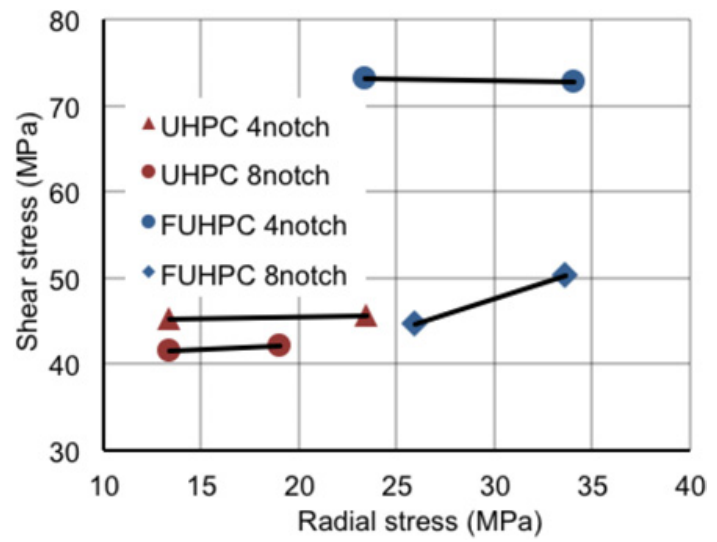

Figure 5. Results from quasi-static shear tests performed on UHPC without additional fibers and UHPC with $2 \%$ volumetric addition of short steel fibers for both four-notch and eight-notch samples representing double shear stress peak behavior with their corresponding radial stress.

the second stress peak has been observed in both series of samples. However, samples with steel fiber reinforcement have shown a larger post peak shear stress and a more gradual decrease towards the same residual stress than in samples with no addition of steel fibers. A possible explanation is that after the second peak, the pull-out strength of fibers is still active. 


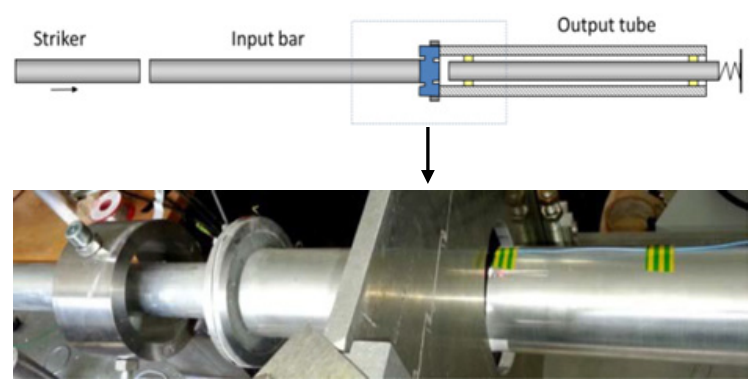

Figure 6. Experimental device used in 3SR Laboratory to perform dynamic PTS tests.

Points representing both first and second shear stress peaks with their corresponding radial stress, summarizing the results from quasi-static experiments are reported in Fig. 5.

A lower overall shear stress is noted for samples with eight-notches, apparently due to the presence of self-confinement of the peripheral part in four-notch samples causing higher shear strength. This is especially observed in fibered four-notch samples. The possible explanation is that the crack-bridging effect resulting from the presence of fibers may probably increase the self-confinement, which cannot be explicitly measured, resulting in higher shear strength in fibered samples with four-notch compared to eight-notch. It can be concluded that it is necessary to practice eight radial notches on ultrahigh performance concrete samples due to the presence of an additional apparent self-confinement.

\section{Dynamic shear testing}

\subsection{Principle of experimental procedure}

The dynamic experimental setup built in 3SR Laboratory is presented on Fig. 6. The sample is subjected to dynamic shear load using a modified Split Hopkinson Pressure Bar (SHPB). It is composed of a projectile, input bar and output tube all made out of the same aluminum alloy. The cylindrical Hopkinson bar and the tube are both instrumented with strain gauges located in their halflengths which purpose is to register wave propagation in the bars due to projectile impact.

A pressure tank is used to launch the striker at impactspeeds up to $20 \mathrm{~m} / \mathrm{s}$ that generates an incident compression pulse in the input bar. The strain gauge measurements on the input bar provide the incident and reflected waves, so the input and output forces between the specimen and the Hopkinson bars are obtained as following:

$$
\begin{aligned}
& F_{\text {input }}=S_{\text {input }} E_{\text {bar }}\left(\varepsilon_{\text {incident }}-\varepsilon_{\text {reflected }}\right) \\
& F_{\text {output }}=S_{\text {output }} E_{\text {bar }}\left(\varepsilon_{\text {transmited }}\right) .
\end{aligned}
$$

Finally, the shear stress in the ligament is deduced by averaging the input and output forces:

$$
\sigma_{\text {shear }}=\frac{F_{\text {input }}+F_{\text {output }}}{2 . S_{\text {ligament }}} .
$$

The axial displacement of the sample middle part is obtained by integrating the particle velocity measured by

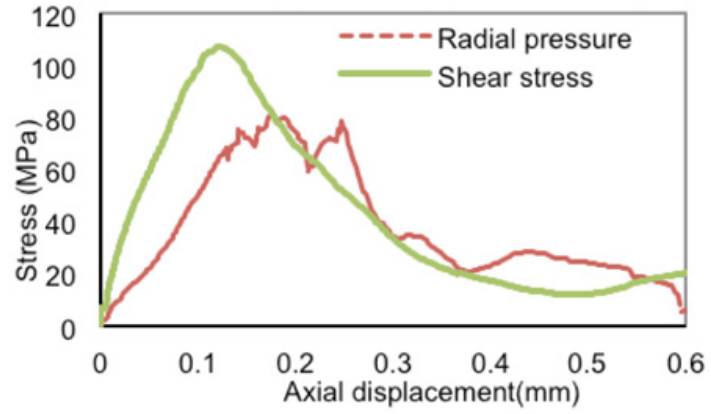

(a)

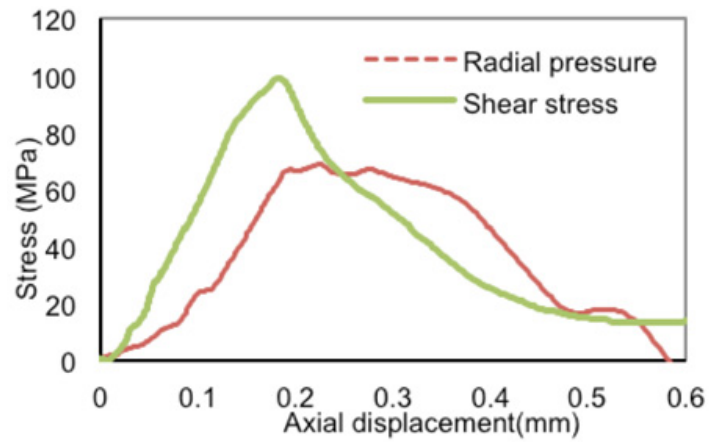

(b)

Figure 7. Results of dynamic shear tests performed on UHPC without additional fibers: (a) four-notch sample (b) eight-notch sample.

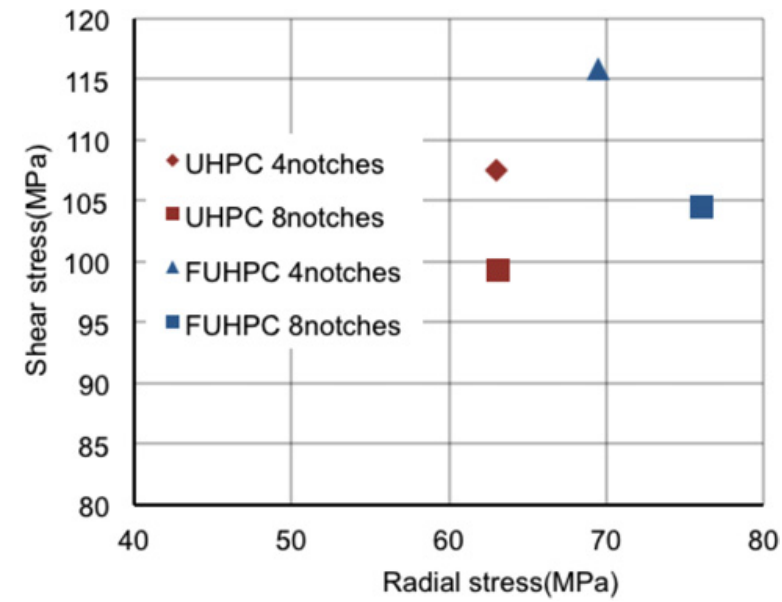

Figure 8. Results of dynamic shear tests performed on UHPC without additional fibers and UHPC with $2 \%$ volumetric addition of short steel fibers for both four-notch and eight-notch samples representing both shear stress peaks with their corresponding radial stress.

the laser interferometer pointed out toward the middle of the output tube.

$$
V_{\text {axial }}=V_{\text {reflected }}+V_{\text {incident }}-V_{\text {transmited }} .
$$

\subsection{Experimental results}

A series of experiments have been carried out on ultra high performance concrete with and without addition of short steel fibers. As in quasi-static experimental procedure, first the samples with four equally distributed radial notches 
have been tested and then, eight notches have been performed in order to avoid self-confinement as explained in previous part. The results of the dynamic shear tests on non-reinforced samples are reported in Fig. 7.

The maximum shear stress reached in four-notch sample without addition of steel fibers was $105^{\circ} \mathrm{MPa}$ while on the other hand, the sample with fibers exhibited higher shear strength of about $116^{\circ} \mathrm{MPa}$. The maximum shear stress reached in non-fiber reinforced eight-notch sample was around $100 \mathrm{MPa}$ while the fibered sample exhibited a shear stress of $108^{\circ} \mathrm{MPa}$ which again is fairly similar.

\subsection{Discussion of the dynamic experiments}

Points representing shear stress peaks with their corresponding radial stress summarizing the results from dynamic tests are reported in Fig. 8. Higher shear strength is observed in comparison to quasi-static results. This is due to a much higher radial stress reached in the ligament. Similar to quasi-static experiments, a decrease of shear strength is observed by increasing the number of radial notches, indicating a presence of apparent self-confinement especially in fiber reinforced samples. However, in this case only one-stress peak behaviour is observed. A possible explanation may be that the presence of tensile cracking prior to pure Mode II is being delayed, therefore affecting the radial stress in the ligament. This, furthermore, could be explained by a higher tensile strength observed in concrete like materials when subjected to high strain rate tensile loading, that has been reported in literature by several authors [8-10].

\section{Conclusion}

A series of PTS experiments have been conducted on an ultra-high performance concrete in quasi-static and dynamic regimes in order to investigate the influence of strain rate on the confined shear strength. Furthermore, half of the samples contained additional short steel fibers in order to study their influence on material behaviour. At the beginning, four radial notches have been performed to reduce the influence of self-confinement. The obtained results in quasi-static testing showed a major difference in shear strength depending on the fiber content. Practicing additional notches, eight in total, and testing the samples in the same regime, resulted in significantly lower shear strength, especially in fibered concrete. A clear positive influence of fibers until the first stress peak has been reported, that reflects in the closure of tensile cracks (crackbridging effect) prior to mode II cracking, increasing the radial stress and consequently influencing the overall shear strength. Moreover, the samples with addition of steel fibers showed a higher post-peak shear stress. Dynamic test results showed much higher shear strength compared to quasi-static results. This difference is thought to be the consequence of a higher radial confinement that could be induced by adjournment of tensile cracking prior to Mode II cracking occurring in the ligament, as tensile strength is substantially increased at high strain rates.

This work has been partially supported by the CEA (French Alternative Energies and Atomic Energy Commission) - Centre de Gramat and by the LabEx Tec 21 (Investissements d'Avenir grant agreement $n^{\circ}$ ANR-11-LABX-0030).

\section{References}

[1] Q. M. Li, S. R. Reid, H. M. Wen, and A. R. Telford, Int. J. Impact Eng. 32, 224-284, (2005)

[2] P. Forquin and F. Hild, J. Eng. Mech. 4, 302-315, (2008)

[3] J. Watkins, Int. J. Fract. 23, 135-138, (1983)

[4] O. Montenegro, D. Sfer, C. M. López, and I. Carol, Proceedings FraMCoS-8, (2013)

[5] P. Forquin, G. Gary, and F. Gatuingt, Int. J. Impact Eng. 35, 425-446, (2008)

[6] P. Forquin, These Doctorat, LMT Cachan, (2003)

[7] P. Forquin, Appl. Mech. Mater. 82, 148-153, (2011)

[8] F. Toutlemonde, C. Boulay, and P. Rossi, Proceedings of FRAMCOS-2, (1995)

[9] J. Weerheijm and J. C. a. M. Van Doormaal, Int. J. Impact Eng. 34, 609-626, (2007)

[10] B. Erzar and P. Forquin, Exp. Mech. 50, 941-955, (2010) 